\title{
The Auslander-Reiten Components in the Rhombic Picture
}

\author{
Markus Schmidmeier and Helene R. Tyler \\ Department of Mathematical Sciences \\ Florida Atlantic University \\ 777 Glades Road \\ Boca Raton, Florida 33431 \\ Department of Mathematics \\ Manhattan College \\ 4513 Manhattan College Parkway \\ Riverdale, New York 10471 \\ markus@math.fau.edu \\ helene.tyler@manhattan.edu \\ Dedicated to Mark Kleiner on the occasion of his 65th birthday
}

\begin{abstract}
For an indecomposable module $M$ over a path algebra of a quiver of type $\widetilde{\mathbb{A}}_{n}$, the Gabriel-Roiter measure gives rise to four new numerical invariants; we call them the multiplicity, and the initial, periodic and final parts. We describe how these invariants for $M$ and for its dual specify the position of $M$ in the Auslander-Reiten quiver of the algebra.

MSC 2010: 16G70 (primary), 05E10, 16D70, 16G20

Keywords: Auslander-Reiten quiver, Auslander-Reiten sequence, GabrielRoiter measure, rhombic picture, string algebra
\end{abstract}

\section{INTRODUCTION}

1.1. The Gabriel-Roiter Measure. We revisit the traditional partition of the module category of a tame hereditary algebra into the preprojective, regular, and preinjective components [2], motivated by recent developments in the Gabriel-Roiter theory. In particular, the Gabriel-Roiter measure of a module, $\mu(M)$, and the dually defined comeasure, $\mu^{*}(M)$, specify the coordinates of the module in the rhombic picture [9]. We focus primarily on tame hereditary $k$-algebras of type $\widetilde{\mathbb{A}}_{n}$, where $k$ is an algebraically closed field, since their modules and homomorphisms are controlled by the combinatorics of strings and bands [3, 7]. This allows us to build a "Greedy Algorithm" for computing the Gabriel-Roiter measure and comeasure for a $k \widetilde{\mathbb{A}}_{n}$-module. We show that the Gabriel-Roiter measure of a $k \widetilde{\mathbb{A}}_{n}$-module $M$ decomposes into three distinct parts:

$$
\mu(M)=\operatorname{init}(M) \cdot \operatorname{per}(M)^{\operatorname{mult}(M)} \cdot \operatorname{fin}(M),
$$

and we extract a great deal of information from this decomposition. To begin, we find that the decomposition identifies the type of the Auslander-Reiten component in which the module resides.

Proposition 1.1. Let $M$ be an indecomposable $k \widetilde{\mathbb{A}}_{n}$-module. 
(1) $M$ is preprojective if and only if $\operatorname{fin}(M)<\operatorname{per}(M)$ and $\operatorname{fin}(D M)>$ $\operatorname{per}(D M)$

(2) $M$ is preinjective if and only if $\operatorname{fin}(M)>\operatorname{per}(M)$ and $\operatorname{fin}(D M)<$ $\operatorname{per}(D M)$

(3) $M$ is regular if and only if $\operatorname{fin}(M)<\operatorname{per}(M)$ and $\operatorname{fin}(D M)<$ $\operatorname{per}(D M)$

Throughout the paper we freely use the Gabriel-Roiter theory terminology introduced by Ringel in [9], and the usual representation theory terminology introduced in [8] (see also the books [2] and [1]). In particular, we recall from [8] that the regular component $\operatorname{Reg}(A)$ of the Auslander-Reiten quiver $\Gamma(A)$ of a tame hereditary $k$-algebra $A$ of Euclidean type is a disjoint union of a $\mathbb{P}_{1}(k)$-family of stable tubes.

1.2. Module Families in the Rhombic Picture. Assume that $k$ is an algebraically closed field and $A$ is a tame hereditary algebra of Euclidean type. We recall from [8] that a regular $A$-module $M$ defines a ray and a coray in its tube. We order the modules on the intersection of the ray and the coray by size, obtaining the family $\left(M_{i}\right)_{i \in \mathbb{N}}$ of $M$. For preprojective and preinjective modules we use the cyclic structure of $\widetilde{\mathbb{A}}_{n}$ to define corresponding families. We define the rhombic limit of $M$ as

$$
\vec{\rho}(M)=\left(\lim _{i \rightarrow \infty} \mu\left(M_{i}\right), \lim _{i \rightarrow \infty} \mu\left(D M_{i}\right)\right)
$$

We will see in Corollary 4.8 and Proposition 4.13 that preprojective and regular families approach the GR-limit from below, while preinjective families approach their GR-limit from above. This can be expressed in terms of chain conditions on totally ordered sets of GR-measures.

Definition 1.2. We call a family $\mathcal{G}$ of GR-measures noetherian (artinian) if $(\mathcal{G},<)$ satisfies the ascending (decending) chain condition. For $\mathcal{M}$ a class of modules, we write

$$
\mathcal{G}(\mathcal{M})=\{\mu(M) \mid M \in \mathcal{M}\}, \quad \mathcal{G}^{*}(\mathcal{M})=\left\{\mu^{*}(M) \mid M \in \mathcal{M}\right\}
$$

for the sets of GR-measures and co-measures of modules in $\mathcal{M}$.

A recent result by Dung and Simson [6, Theorem 3.2] states that a right artinian ring $R$ is right pure semisimple if and only if the set $\mathcal{G}($ ind $R$ ) is noetherian. We obtain in our situation:

Corollary 1.3. Let $\mathcal{P}, \mathcal{R}, \mathcal{Q}$ be full sets of representatives of indecomposable preprojective, regular and preinjective modules over a path algebra of type $\widetilde{\mathbb{A}}_{n}$. Then the sets $\mathcal{G}(\mathcal{P}), \mathcal{G}(\mathcal{R})$ and $\mathcal{G}^{*}(\mathcal{P})$ are artinian but not noetherian, and the sets $\mathcal{G}(\mathcal{Q}), \mathcal{G}^{*}(\mathcal{R}), \mathcal{G}^{*}(\mathcal{Q})$ are noetherian but not artinian. 
The concept of limits is compatible with Auslander-Reiten sequences:

TheOREM 1.4. Let $0 \rightarrow A \rightarrow B_{1} \oplus B_{2} \rightarrow C \rightarrow 0$ be an AuslanderReiten sequence such that the middle term consists of two indecomposable summands. Then the rhombic limits of $A, B_{1}, B_{2}, C$ form a (possibly degenerate) parallelogram in the rhombic picture. Moreover, the nondegenerate sides of the parallelogram are parallel to the $\mu$ and $\mu^{*}$ axes.

1.3. The Tiling Theorem. Finally we present a result that investigates the phenomenon that for a given tube $\mathcal{T}$, the sequence in which the modules on a ray or on a coray occur can be read off from the arrangement of their limit points in the rhombic picture. Namely, we have seen in Theorem 1.4 that for the modules on any ray in a given tube $\mathcal{T}$, the GR-limits in the rhombic picture lie on a line $\ell$ which is parallel to the $\mu^{*}$-axis. Under certain conditions on $Q$ (we say " $Q$ has a widest hill"), the precise sequence of module families on the ray can be read off from the rhombic picture by just repeating the sequence in which their limits occur on $\ell$. By combining this result with the corresponding statement for corays, we obtain that the arrangement of limit points in the rhombic picture for the families in $\mathcal{T}$ represents the sequences in which those families occur along the rays and corays in $\mathcal{T}$. In this sense, the arrangement of limit points in the rhombic picture "provides a tiling for the tube".

Let $M$ be a regular module, sufficiently large so that each part of its measure

$$
\mu(M)=\operatorname{init}(M) \cdot \operatorname{per}(M)^{\operatorname{mult}(M)} \cdot \operatorname{fin}(M)
$$

is defined. Then the periodic part, $\operatorname{per}(M)$, which may be of type $\mathcal{L}$, $\mathcal{R}$ or $(h)$, distinguishes the tube containing $M$. More precisely, for a fixed orientation of the quiver $Q$, the sequences $\mathcal{L}$ and $\mathcal{R}$ consist of left and right hooks, respectively, which determine the orientation on the quiver, while $h=n+1$ is the dimension of a quasisimple homogeneous module. The integer mult $(M)$ then gives the position of $M$ within its family. The remaining "waist-free" part of the measure

$$
\operatorname{wf}(M)=\operatorname{init}(M) \cdot \operatorname{fin}(M)
$$

describes the position of the family within the tube. This is an invariant of the family, as the waist-free parts are totally ordered. In special cases, this ordering agrees with the ordering of the rays within the tube. In section 6.5 we will give a precise definition of a "widest valley" and "widest hill" for an $\widetilde{\mathbb{A}}_{n}$ quiver. 
THEOREM 1.5. Suppose the quiver $Q$ of type $\widetilde{\mathbb{A}}_{n}$ has a unique widest valley and a unique widest hill. Then for each tube, the system of limits in the rhombic picture provides a tiling for the tube.

We give an example to illustrate its statement.

ExAmple 1.6. We consider one of the exceptional tubes for the twosink, two-source $\widetilde{\mathbb{A}}_{4}$ quiver.

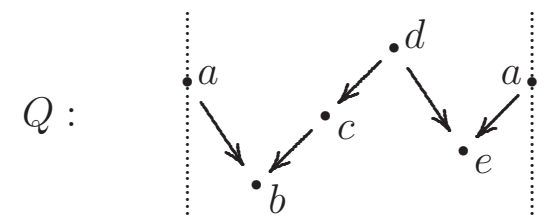

We will see that the quiver has indeed a unique widest valley and a unique widest hill.

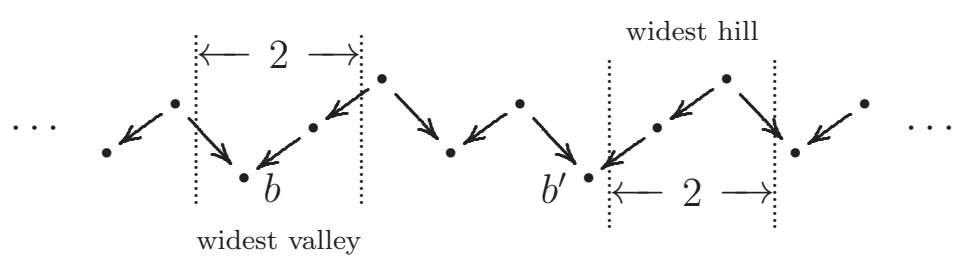

Here we draw the tube with its mouth at the top, to more easily display the correspondence between the arrangement of the rhombic limits and of the AR-sequences in the tube.
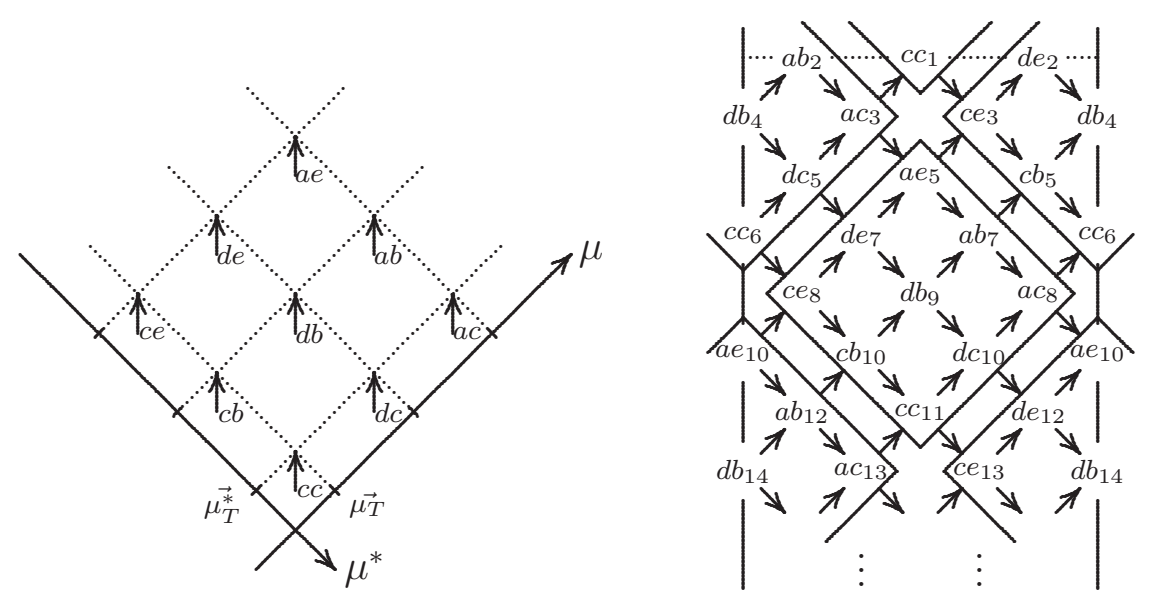

1.4. Organization of this paper. In Section 2 we review and slightly modify the basic terminology about the Gabriel-Roiter measure. In particular, the sequences defining the measure in [9] are obtained from the sequences that we will use by taking partial sums. We also adapt terminology for dealing with string and band modules to the situation where the underlying quiver is of type $\widetilde{\mathbb{A}}_{n}$ and carries a fixed orientation. 
Many of the results in our paper will be obtained through a careful analysis of the Greedy Algorithm, which we define and discuss in Section 3. With this tool we can decompose the Gabriel-Roiter measure of a module into initial, periodic and final parts.

The special shape of the quiver of type $\widetilde{\mathbb{A}}_{n}$ allows us to define in Section 4 the family $\left(M_{i}\right)$ of a string module $M$ as the modules on the intersection of the ray and the coray (or the two rays or corays) given by $M$. We characterize the components of the Auslander-Reiten quiver in terms of properties of the limits of the GR-measures of the modules $M_{i}$ and $D M_{i}$ and obtain a proof for Proposition 1.1.

In Section 5 we present a general result. There we consider families of modules in a stable tube for an arbitrary algebra. We show that in the rhombic picture, the modules in a family approach the rhombic limit from below and give the proof for Theorem 1.4.

In the final Section [6 we return to the case where the quiver has type $\widetilde{\mathbb{A}}_{n}$. We distinguish the tubes in terms of the periodic parts of the GRmeasures of their modules. For each tube, we refine the rhombic limit to make the parallelogram in Theorem 1.4 non-degenerate and show that for suitable quivers, the system of limit points in the rhombic picture gives rise to a tiling of the tube.

\section{Preliminaries}

2.1. The Gabriel-Roiter Measure. The Gabriel-Roiter measure of a module was introduced by Ringel in [9]. There, one considers all possible chains of indecomposable submodules and imposes an ordering on the lists of their lengths. Here, we begin by making a slight modification to Ringel's definition. Namely, instead of recording the lengths of the modules in an indecomposable filtration, we list the lengths of the subsequent quotients. The ordering of the measures remains the same as Ringel's. We recall the details.

Let $\mathcal{F}$ denote the set of all (finite or infinite) sequences $\left(a_{i}\right)$ with entries in $\mathbb{N}=\{1,2, \ldots\}$. Define a total ordering on $\mathcal{F}$ by putting $\left(a_{i}\right)<_{\mathcal{F}}\left(b_{i}\right)$ if either $\left(a_{i}\right)$ is a proper subsequence of $\left(b_{i}\right)$ or if there exists $\ell \in \mathbb{N}$ with $a_{i}=b_{i}$ for $i<\ell$ and $a_{\ell}>b_{\ell}$. Note that with this ordering, $\mathcal{F}$ is a compact and complete metric space. Consider the map $e: \mathcal{F} \rightarrow \mathbb{R}$ given by $\left(a_{i}\right) \mapsto \sum_{\ell} 2^{-\sigma_{\ell}}$ where $\sigma_{\ell}=\sum_{i \leq \ell} a_{i}$ is the partial sum. Then $\left(a_{i}\right)<_{\mathcal{F}}\left(b_{i}\right)$ in $\mathcal{F}$ if and only if $e\left(a_{i}\right)<e\left(b_{i}\right)$ in $\mathbb{R}$ unless one of the sequences is infinite and has almost all entries equal to one.

Now let $\Lambda$ be an artin algebra and let $M$ be a $\Lambda$-module. A sequence $0=M_{0} \subsetneq M_{1} \subsetneq \cdots$ of submodules of $M$ where each $M_{i}$ with $i>$ 
0 is indecomposable and of finite length is called an indecomposable filtration for $M$. The GR-measure $\mu(M)$ is defined as the supremum taken in $\mathcal{F}$ of the sequences $\left(\left|M_{i} / M_{i-1}\right|\right)$ where $0=M_{0} \subsetneq M_{1} \subsetneq \ldots$ is an indecomposable filtration for $M$. Whenever the supremum is attained, this chain is called a GR-filtration. As noted in [9], if $M$ is finitely generated, then a GR-filtration exists if and only if $M$ is indecomposable.

Dually we denote by $\mathcal{F}^{*}$ the set $\mathcal{F}$ with the opposite ordering, so $\left(a_{i}\right)<_{\mathcal{F}}^{*}\left(b_{i}\right)$ holds if and only if $\left(a_{i}\right)>_{\mathcal{F}}\left(b_{i}\right)$. We define an indecomposable cofiltration for $M$ as a sequence $\cdots M_{2} \subsetneq M_{1} \subsetneq M_{0}=M$ where each factor $M / M_{i}$ for $i \geq 1$ is indecomposable of finite length. Then the $G R$-comeasure $\mu^{*}(M)$ is the infimum of the sequences $\left(\left|M_{i-1} / M_{i}\right|\right)$ where the $M_{i}$ form an indecomposable cofiltration for $M$, and where the infimum is taken in $\mathcal{F}^{*}$. Clearly, if $M$ is a finite dimensional module then $\mu^{*}(M)=\mu(D M)$. Here $D M$ is the dual module, which is defined over the opposite algebra.

2.2. String Modules for $\widetilde{\mathbb{A}}_{n}$. For an arbitrary quiver $Q$, the GRmeasure of a $k Q$-module may be quite difficult to compute. But as we will see, when $Q$ is a quiver of type $\widetilde{\mathbb{A}}_{n}$, the string algebra structure of $k Q$ (see, for example, [3] and [7]) allows us to construct an explicit algorithm for computing GR-measures. For such a quiver $Q$, let $f: \widetilde{Q} \rightarrow Q$ be a universal covering, so that $\widetilde{Q}$ is a quiver of type $\mathbb{A}_{\infty}^{\infty}$ with vertex set $\mathbb{Z}$ and the orientation of the arrow between $i$ and $i+1$ is given by the arrow between $f(i)$ and $f(i+1)$. Note that by fixing a covering and labeling the vertices in $\widetilde{Q}$ with integers, we have implicitly chosen an orientation for $\widetilde{\mathbb{A}}_{n}$. Recall that a string module is determined by its starting point and length. Thus, the covering $f$ induces a one-to-one correspondence between string modules for $k Q$ and intervals in $\widetilde{Q}$, up to the shift by a fundamental domain. Moreover, the Auslander-Reiten component to which an indecomposable module belongs is completely determined by its string or band structure. We call a string preprojective (preinjective, left regular, right regular) if in the corresponding interval in $\widetilde{Q}$ the two arrows in $\widetilde{Q}$ neighboring the interval but lying outside of it point towards the interval (away from the interval, to the left, to the right). Then the preprojective modules, the preinjective modules, and the modules in the two exceptional tubes are string modules corresponding to preprojective, preinjective, left regular, and right regular strings. The band modules lie in homogeneous tubes. Each string is the domain of at most two irreducible morphisms, corresponding to the endpoints of the interval. At each 
endpoint we can perform at most one of the following two operations. If there is an incoming arrow at an endpoint, we can " add a hook", by expanding the string to the next incoming arrow. If there is an outgoing arrow at an endpoint, we "delete a cohook" by contracting the string to the endpoint of the previous outgoing arrow. As a consequence, all irreducible maps in the preprojective (preinjective) component are monomorphisms (epimorphisms) since all of the maps are formed by adding hooks (deleting cohooks).

\section{Structure of GR-Measures for $\widetilde{\mathbb{A}}_{n}$}

3.1. The Greedy Algorithm for $\widetilde{\mathbb{A}}_{n}$ Strings. Now suppose that $M$ is a string module and that $\mathcal{M}: 0=M_{0} \subsetneq M_{1} \subsetneq \cdots \subsetneq M_{n}=M$ is a GR-filtration, such that each $M_{i}$ is a string module. This is the case for preprojective modules and for non-homogeneous regular modules. Then there is a sequence of intervals $\emptyset=I_{0} \subsetneq I_{1} \subsetneq \cdots \subsetneq I_{n}=I$ in $\widetilde{Q}$ corresponding to the filtration $\mathcal{M}$. Since $\mathcal{M}$ defines the GRmeasure, it follows that $I_{1}$ consists only of a sink in $I$, and each $I_{i+1}$ is obtained from $I_{i}$ by adding a left hook or a right hook in $I$. Bearing this correspondence in mind, we now present the "Greedy Algorithm", which provides an efficient tool for the computation of the GR-measure of $M$.

(1) Begin by choosing a sink $\sigma$ in $I$. Thus, the first entry in the measure is (1).

(2) Next, compute the sequences $\left(\lambda_{i}\right)$ and $\left(\rho_{i}\right)$ of lengths of left hooks and right hooks, respectively, starting at the sink $\sigma$.

(3) If $\left(\lambda_{i}\right)<_{\mathcal{F}}\left(\rho_{i}\right)$, add $\rho_{1}$ to the measure and delete the first entry in the sequence $\left(\rho_{i}\right)$. If the inequality is reversed, add $\lambda_{1}$ to the measure and delete this entry in $\left(\lambda_{i}\right)$. If $\left(\lambda_{i}\right)=\left(\rho_{i}\right)$, we may make the choice freely.

(4) Repeat (3) until both sequences $\left(\lambda_{i}\right)$ and $\left(\rho_{i}\right)$ are exhausted. This is the highest measure based on the starting point $\sigma$.

(5) Repeat (1-4) until each sink in $I$ has been used as a starting point. The supremum of the resulting sequences is, therefore, the GR-measure of $M$.

Example: Consider the quiver $Q$ of type $\widetilde{\mathbb{A}}_{4}$ from Example 1.6. and the string module $c e_{18}$, as pictured.

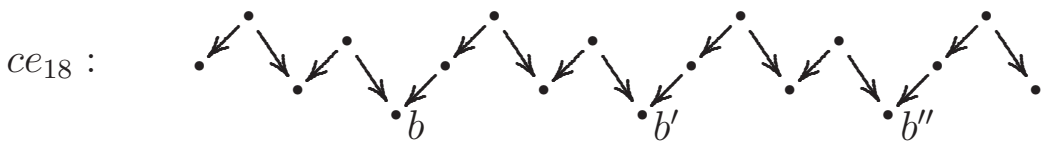


Note that the module $c e_{18}$ is regular and occurs in an exceptional tube. Thus any submodule of $c e_{18}$ is either preprojective, or occurs in the same tube. In each case, the submodule is a string module. Hence, the Greedy Algorithm will produce the GR-measure. Moreover, we need only run the algorithm beginning from sinks that lie in "widest valleys", as this will maximize the small entries at the beginning of the measure. Then running the algorithm, we find the following.

\begin{tabular}{c|c|c|c}
$\begin{array}{c}\text { starting } \\
\text { point }\end{array}$ & $\begin{array}{c}\text { left hook } \\
\text { sequence }\left(\lambda_{i}\right)\end{array}$ & $\begin{array}{c}\text { right hook } \\
\text { sequence }\left(\rho_{i}\right)\end{array}$ & $\begin{array}{c}\text { candidate for } \\
\text { GR-measure }\end{array}$ \\
\hline$b$ & $(2,2)$ & $(1,2,2,1,2,2,1,2)$ & $(1,1,2,2,1,2,2,1,2,2,2)$ \\
$b^{\prime}$ & $(2,3,2,2)$ & $(1,2,2,1,2)$ & $(1,1,2,2,1,2,2,3,2,2)$ \\
$b^{\prime \prime}$ & $(2,3,2,3,2,2)$ & $(1,2)$ & $(1,1,2,2,3,2,3,2,2)$
\end{tabular}

Comparing the candidates generated by each starting point, we find that $\mu\left(c e_{18}\right)=(1,1,2,2,1,2,2,1,2,2,2)$.

Looking back at the example, we notice that the GR-measure $\mu\left(c e_{18}\right)$ has a periodic part in the center. In fact, depending on the starting point of the period, there are several choices for a decomposition of the measure into an initial part, a periodic part and a terminal part:

$$
\begin{aligned}
\mu\left(c e_{18}\right)=(1,1,2,2,1,2,2,1,2,2,2) & =(1 ; 122-122-122 ; 2) \\
& =(11 ; 221-221 ; 222) \\
& =(112 ; 212-212 ; 22)
\end{aligned}
$$

This partitioning of the GR-measure is typical, as we will see in the next section. Moreover, we will see that there is a canonical choice for the periodic part of the measure.

3.2. The Periodic Part. For a finite sequence of natural numbers $\mathcal{K}=\left(k_{1}, \ldots, k_{s}\right)$, we denote by $\mathcal{K}_{u}=\left(k_{u+1}, k_{u+2}, \ldots, k_{u}\right)$ the rotation by $u$ where the subscripts are taken modulo $s$.

LEMMA 3.1. Let $\mathcal{K}=\left(k_{1}, \ldots, k_{s}\right)$ be a finite sequence of natural numbers such that

(1) $\mathcal{K}$ is minimal in $\mathcal{F}$ among its rotations $\mathcal{K}_{u}$ and

(2) $\mathcal{K}$ is not a power of a proper subsequence.

Then for each $1 \leq u \leq s-1$, the tail $\left(k_{u+1}, \ldots, k_{s}\right)$ of $\mathcal{K}$ is strictly larger in $\mathcal{F}$ than $\mathcal{K}$.

Proof. Suppose that $\left(k_{u+1}, \ldots, k_{s}\right)<_{\mathcal{F}} \mathcal{K}$ for some $1 \leq u \leq s-1$. Together with the minimality of $\mathcal{K}$ among the $\mathcal{K}_{i}$ we obtain

$$
\left(k_{u+1}, \ldots, k_{s}\right)<_{\mathcal{F}}\left(k_{1}, \ldots, k_{s}\right)<_{\mathcal{F}}\left(k_{u+1}, \ldots, k_{u}\right),
$$


so $k_{1}=k_{u+1}, \ldots, k_{s-u}=k_{s} \quad(*)$.

We show by induction on $\ell=1, \ldots, s$ that $k_{i}=k_{s-u+i}$ holds for each $1 \leq i \leq \ell$. The inequality $\mathcal{K}<_{\mathcal{F}} \mathcal{K}_{u}$ together with $(*)$ and the induction assumption yields that $k_{s-u+\ell} \geq k_{\ell}$. The inequality $\mathcal{K}<_{\mathcal{F}} \mathcal{K}_{s-u}$ together with the induction assumption implies $k_{\ell} \geq k_{s-u+\ell}$, which proves the claim.

Since $\mathcal{K}$ is not a power of a proper subsequence, we deduce that $u \equiv 0$ $\bmod s$, finishing the proof of the Lemma.

We apply this lemma to the sequences $\mathcal{L}=\left(\ell_{i}\right)_{i=1, \ldots, s}$ and $\mathcal{R}=\left(r_{i}\right)_{i=1, \ldots, t}$ of lengths of left hooks and of right hooks in $\widetilde{Q}$, respectively, covering in each case one period (the quiver $Q$ may consist of several periods). We assume that $\mathcal{L}$ and $\mathcal{R}$ are rotated such that they are minimal among the $\mathcal{L}_{u}$ and $\mathcal{R}_{u}$, respectively. The lemma then reveals a useful, albeit counterintuitive, fact. If we begin with a minimally rotated hook sequence and "bite off" a hook from the beginning of the sequence, the remaining partial hook sequence is larger in $\mathcal{F}$ than the complete one. We now see what is so greedy about our algorithm. According to Lemma 3.1, once the first hook has been taken from, say, $\mathcal{R}$, all subsequent hooks from $\mathcal{R}$ and its full iterations will be taken.

Note now that $Q$ is symmetric if and only if $\mathcal{L}=\mathcal{R}$ (since each of the sequences determines and is determined by $\widetilde{Q}$ ). If $Q$ is not symmetric, one direction is distinguished.

Definition 3.2. Let $Q$ be an asymmetric quiver and suppose that $\mathcal{L}$ and $\mathcal{R}$ are its mimimally rotated left and right hook sequences. We say the take-off direction is to the right (left) if $\mathcal{L}<_{\mathcal{F}} \mathcal{R}\left(\mathcal{R}<_{\mathcal{F}} \mathcal{L}\right)$.

Thus, whenever the Greedy Algorithm can choose between a sequence of left hooks starting with $\mathcal{L}$ and a sequence of right hooks starting with $\mathcal{R}$, then it will move in the take-off direction. In light of this, we introduce the following convention for the reminder of this paper. From now on we will assume that either $Q$ is symmetric or that the orientation is chosen such that $\mathcal{L}<_{\mathcal{F}} \mathcal{R}$ holds. That is, if $Q$ is not symmetric, we assume without loss of generality that its take-off direction is to the right. In the next section, we will see that the GR-measure has a characteristic decomposition, in which these hook sequences play and important role. Exactly one of the hook sequences may appear strategically in a module's measure, and for a regular module this will distinguish the tube in which it resides. Moreover, the exceptional tubes behave distinctly with respect to the partition of the module category into the take-off, central, and landing parts. 
EXAMPLE 3.3. Returning to the example in the previous section, the right hooks have lengths $1,2,2$, and the left hooks have lengths 3,2 . Hence, the hook length sequences that are minimal among their rotations are $\mathcal{R}=(2,2,1)$ and $\mathcal{L}=(3,2)$. We see that the take-off direction is to the right and that the canonical choice for the decomposition is $\mu\left(c e_{18}\right)=(1,1 ; 2,2,1,2,2,1 ; 2,2,2)$.

3.3. The IPF Decomposition of the GR-Measure. We now show that the GR-measure $\mu$ of a module $M$, with $\operatorname{dim} M$ sufficiently large, consists of three distinct parts: an initial part, a periodic part, and possibly a final part. That is,

$$
\mu(M)=\operatorname{init}(M) \cdot \operatorname{per}(M)^{\operatorname{mult}(M)} \cdot \operatorname{fin}(M)
$$

Here we use multiplicative notation to indicate concatenation of sequences. We will see that the periodic part of the GR-measure may be of type $\mathcal{L}, \mathcal{R}$ or $(h)$ where $h=\left|Q_{0}\right|$.

Suppose that $\mathcal{M}: 0=M_{0} \subsetneq M_{1} \subsetneq \cdots \subsetneq M_{n}=M$ is a GR-filtration of $M$. We call the module $M_{n-1}$ a $G R$-submodule of $M$. Note that $\mu(M)=\mu\left(M_{n-1}\right) \cdot\left(\operatorname{dim} M / M_{n-1}\right)$. We first consider modules $M$ such that the GR-submodule of $M$ is a string module. This includes all preprojective modules and all non-homogeneous regular modules. We have two cases to consider.

Case 1: The hook length sequences $\mathcal{L}$ and $\mathcal{R}$ are not equal. Recall that we assume then that the take-off direction is to the right. Given the starting point $b$, a candidate for the GR-measure produced by the Greedy Algorithm has the following form:

(1) First, there is an initial part. It consists of a leading 1, some of the $\lambda_{i}$ 's before the first occurance of $\mathcal{L}$ to the left of $b$, and all $\rho_{j}$ before the first occurance of $\mathcal{R}$ to the right of $b$. Note that the length of the initial part is at most $s+t-1$.

(2) Next, according to Lemma 3.1, there is a periodic part consisting of all full iterations of $\mathcal{R}$ occurring to the right of $b$.

(3) At this point, there may occur some of the remaining parts of $\left(\rho_{j}\right)$, and all the remaining $\lambda_{i}$ before the start of the first $\mathcal{L}$.

(4) Lemma 3.1 now tells us that once the first hook is taken from $\mathcal{L}$, all subsequent hooks from $\mathcal{L}$ and its full iterations will be taken.

(5) In the end, there may be a final part.

Now we vary the starting point:

If the measure of the part starting at (3) above is less than $\mathcal{R}$, we obtain a larger measure by moving the starting point by one period to the left, 
thus expanding the part in (2) at the expense of (4). This process can be repeated until no copy of $\mathcal{L}$ remains in the measure. Conversely, if the measure of the part starting at (3) above is larger than $\mathcal{R}$, we obtain a larger measure by moving the starting point by one period to the right, expanding (4) at the expense of (2). As a consequence, we obtain that for a GR-measure, either the part in (2) or the part in (4) is empty. Hence the measure $\mu(M)$ consists of an initial part, $\operatorname{init}(M)$, a periodic part, $\operatorname{per}(M)$, which repeats $\operatorname{mult}(M)$ times, and then possibly a final part, $\operatorname{fin}(M)$. That is,

$$
\mu(M)=\operatorname{init}(M) \cdot \operatorname{per}(M)^{\operatorname{mult}(M)} \cdot \operatorname{fin}(M)
$$

Case 2: The hook length sequences $\mathcal{L}$ and $\mathcal{R}$ are equal; i.e., the quiver is symmetric. Then given a starting point $b$, the candidate for measure produced by the Greedy Algorithm has the following form:

(1) There is an inital part, consisting of a leading 1 , and all entries from the sequences $\left(\lambda_{i}\right)$ and $\left(\rho_{j}\right)$ leading up to the first occurance of $\mathcal{L}=\mathcal{R}$.

(2) The central part is periodic consisting of all sequences $\mathcal{R}$ in $\left(\lambda_{i}\right)$ and $\left(\rho_{j}\right)$.

(3) At the end, there may be a final part.

Again, the GR-measure is obtained by choosing a starting point suitably. And, again, the measure decomposes as in the previous case.

Now we consider the situation in which the GR-submodule of $M$ is a band module. Then $M$ contains in its GR-filtration a quasi-simple homogeneous module $H$ [5, Corollary 4.5] or [10, Theorem B].

In [9] it was shown that if the base field is infinite, then $\mu_{H}$ is not a measure of finite type. Hence, $\mu_{H}$ is not a take-off measure. It follows that $\mu_{H}$ exceeds the GR-measure of any preprojective module.

Next we show that a homogeneous module of quasi-length $q$ has measure

$$
\mu(H[q])=\mu_{H} \cdot(h)^{q-1},
$$

where $\left|Q_{0}\right|=h=n+1$. For this we use induction on $q$. The case where $q=1$ has been handled above. Suppose for the induction step that $\mu(H[q])$ is as claimed. Since the only submodules of $H[q+1]$ are either of the form $H[u]$ for some $u \leq q$, or are preprojective and hence have smaller GR-measure, we see that the GR-submodule of $H[q+1]$ is $H[q]$. The claim follows. Note that in this situation the terminal part of the measure is empty. 
It remains for us to compute the measure of a preinjective module. Since all irreducible maps in the preinjective component are epimorphisms, the GR-submodule $X$ of a preinjective module is either preprojective or regular. However, if $X$ is regular, it may be either a string module or a band module. If $X$ is a string module, then the GR-measure of $M$ is computed by the Greedy Algorithm. If $X$ is a band module, then the following lemma is useful.

LEMMA 3.4. Suppose $M$ is a preinjective module of dimension $q h+r$, where $h=\left|Q_{0}\right|, q \geq 1$, and $0<r \leq h-1$. Then there is a (homogeneous) band module $N$ of dimension qh that embeds as a submodule of $M$.

Proof. We begin by showing that there is a non-zero map $g: H=$ $H[1] \rightarrow M$. We know that for some $s$, there is a non-zero map $f$ : $H[s] \rightarrow M$. If $f$ does not vanish on the quasi-socle $H[1]$, then we may take $g$ to be the restriction of $f$ to $H[1]$. If, however, $f$ does vanish on $H[1]$, then $f$ factors over a non-zero map $\tilde{f}: H[s-1] \rightarrow M$. Thus, after at most $s-1$ steps we obtain the desired map $g$. For dimension reasons, $g$ cannot be an epimorphism; hence, $g$ is a monomorphism: Since the image of $g$ is regular, the kernel of $g$ (which has defect 0 ) must be regular. But $H[1]$ being quasi-simple has no non-zero proper regular submodule.

Next, for every $1 \leq s \leq q$, we construct by iteration an embedding $H[s] \rightarrow M$. Having already dealt with the base case, we can assume in the induction step that an inclusion $f: H[s] \rightarrow M$ is given. Since $f$ is not a split monomorphism, it factors over $\left(f^{\prime}, f^{\prime \prime}\right): H[s+1] \oplus$ $H[s-1] \rightarrow M$. Again, since $f^{\prime}$ is not an epimorphism, its image is a regular submodule, and hence the kernel of $f^{\prime}$ is a regular submodule of $H[s+1]$. Since the composition $H[1] \rightarrow H[s] \rightarrow H[s+1] \rightarrow M$ is non-zero, $f^{\prime}$ must be monic.

As the GR-measures of homogeneous modules increase with the dimension of the module, as shown above, it follows that $X$ is of the form $H[q]$ where $q$ is given by $\operatorname{dim} M=q h+r$. In this case, $\mu(M)=\mu_{H} \cdot(h)^{q-1} \cdot(r)$.

In practice, therefore, the GR-measure of a preinjective module is computed as the maximum of the output of the Greedy Algorithm and the measure $\mu_{H} \cdot(h)^{q-1} \cdot(r)$, since it is usually quite difficult to determine whether the GR-submodule is a string or a band. However, we do know the following:

LEMma 3.5. Suppose $M$ is an indecomposable module with GR-filtration $\mathcal{M}: 0=M_{0} \subsetneq M_{1} \subsetneq \cdots \subsetneq M_{n}=M$. If there exists some $i \in$ 
$\{1,2, \ldots, n-1\}$ such that $M_{i}$ is a band module, then the GR-submodule of $M$ is a band module.

Proof. Assume $M_{i}$ is a band module for some $i \in\{1,2, \ldots, n-2\}$. Then there is a monomorphism $f: M_{i} \rightarrow M_{i+1}$. Since an irreducible map ending at a preinjective module must be an epimorphism, we have that $M_{i+1}$ is also homogeneous regular. Repeat the argument until $i+1=n-1$.

The following proposition summarizes the results obtained thus far:

Proposition 3.6. Let $Q$ be a quiver of type $\widetilde{\mathbb{A}}_{n}$ with $\left|Q_{0}\right|=h=$ $n+1$ and let $\mathcal{L}$ and $\mathcal{R}$ denote the minimally rotated left and right hook sequences for $Q$. The GR-measure $\mu$ of a large enough indecomposable $k Q$-module $M$ has the form

$$
\mu(M)=\operatorname{init}(M) \cdot \operatorname{per}(M)^{\operatorname{mult}(M)} \cdot \operatorname{fin}(M) .
$$

The initial part, $\operatorname{init}(M)$, is of length at most $s+t-1$, ending just before the first occurance of a full sequence $\mathcal{L}, \mathcal{R}$ or $(h)$, which then is the periodic part. It occurs with multiplicity $\operatorname{mult}(M) \in \mathbb{N}$. Then, the (possibly empty) final part, $\operatorname{fin}(M)$, is of length at most $s+t-2$.

Corollary 3.7. The comeasure of a large enough indecomposable module $M$ has the form

$$
\mu^{*}(M)=\operatorname{init}(D M) \cdot \operatorname{per}(D M)^{\operatorname{mult}(D M)} \cdot \operatorname{fin}(D M)
$$

Proof. By definition, $\mu^{*}(M)=\mu(D M)$.

REMARK 3.8. The definition of $\operatorname{init}(M), \operatorname{per}(M)$, fin $(M)$ and their duals will be extended in Remark 4.4 to modules that are not large enough to have a non-trivial periodic part.

In light of the corollary, we may define $\operatorname{init}^{*}(M)=\operatorname{init}(D M)$, $\operatorname{per}^{*}(M)=$ $\operatorname{per}(D M), \operatorname{mult}^{*}(M)=\operatorname{mult}(D M), \operatorname{fin}^{*}(M)=\operatorname{fin}(D M)$. Thus we have introduced eight combinatorial invariants for a module $M$. The interactions among them reveal representation-thoretic information about the module. For example, we can determine the Auslander-Reiten component in which the module resides. Such issues are considered in the sections that follow.

\section{Module Families in the Rhombic Picture}

4.1. Rays and Corays. Over the path algebra $K Q$, each indecomposable module $M$ is the starting point and end point of at most two 
irreducible morphisms; hence it lies on two rays or corays. The intersection of the two rays, the two corays, or the ray and the coray defines the family of $M$ as follows.

For this note that as the quiver $Q$ is cyclic, the irreducible morphisms in the preprojective component and in the preinjective component can be partitioned into two classes, clockwise and counterclockwise. Thus, an indecomposable preprojective module lies on two rays given by irreducible monomorphisms, one going in the clockwise direction, the other in the counterclockwise direction. Each ray begins with a projective module. Dually, each indecomposable preinjective module defines two corays given by irreducible epimorphisms, one clockwise, the other counterclockwise, each ending at an injective module.

Each of the regular modules defines a ray of monomorphisms starting at a quasi-simple module, and a coray of epimorphims, ending at a quasi-simple module.

Definition 4.1. Let $M$ be an indecomposable module. The family of $M$ consists of the modules on the intersection of the two rays (if $M$ is preprojective), the two corays (if $M$ is preinjective) or the ray and the coray (if $M$ is regular). We order the modules in the family by dimension.

REMARK 4.2. It is easy to see that two string modules lie in the same family if and only if they have the same string type, in the sense that the strings start at the same vertex and end at the same vertex.

LEMMA 4.3. If $M$ and $M^{\prime}$ are large enough and belong to the same family, then $\operatorname{init}(M)=\operatorname{init}\left(M^{\prime}\right), \operatorname{per}(M)=\operatorname{per}\left(M^{\prime}\right)$, and $\operatorname{fin}(M)=$ fin $\left(M^{\prime}\right)$. That is, $\mu(M)$ and $\mu\left(M^{\prime}\right)$ only may differ in the multiplicity of the periodic part.

Proof. We have seen that for a preprojective or regular string module, the Greedy Algorithm chooses its starting point near one of the ends of the string. Hence, for two modules in the same family, the GRmeasures will differ only in the length of the periodic part. We also have seen that for a preinjective module $M$ the GR-measure is the maximum of the output of the Greedy Algorithm and $\mu_{H} \cdot(h)^{q-1} \cdot(r)$, where $\operatorname{dim} M=q h+r$. In either case, the maximum in $\mathcal{F}$ depends only on the early entries of the sequence. Hence, either both modules have a GR-submodule that is a string module, or both modules have a GR-submodule that is homogeneous. The result follows.

REMARK 4.4. As a consequence of Lemma 4.3, the definition of the initial, periodic and final parts of the GR-measure can now be extended 
to indecomposable modules of arbitrary (small) dimension. Put

$$
\operatorname{init}(M)=\operatorname{init}\left(M_{i}\right), \quad \operatorname{per}(M)=\operatorname{per}\left(M_{i}\right), \quad \operatorname{fin}(M)=\operatorname{fin}\left(M_{i}\right)
$$

if $M_{i}$ occurs in the family of $M$ and is large enough.

4.2. Limits and Colimits. In [9], Ringel introduced the rhombic picture $\mathcal{F} \times \mathcal{F}^{*}$, which provides a visual organization of the module category. There, each module $M$ is given the coordinates $\left(\mu(M), \mu^{*}(M)\right)$. We apply this notion to module families.

LEMmA 4.5. Let $M$ be an indecomposable module and let $\left(M_{i}\right)_{i=1}^{\infty}$ denote the family of $M$. Then $\lim _{i \rightarrow \infty} \mu\left(M_{i}\right)$ and $\lim _{i \rightarrow \infty} \mu^{*}\left(M_{i}\right)$ both exist.

Proof. Note that GR-measures increase along a ray and, likewise, GRcomeasures decrease along a coray. The existence of the limits then follows from the fact that $\mathcal{F}$ is a compact and complete metric space.

Definition 4.6. Let $M$ be an indecomposable module in a stable tube and let $\left(M_{i}\right)_{i=1}^{\infty}$ denote the family of $M$.

(1) The GR-limit of $M$ is $\vec{\mu}(M)=\lim _{i \rightarrow \infty} \mu\left(M_{i}\right)$

(2) The GR-colimit of $M$ is $\overrightarrow{\mu^{*}}(M)=\lim _{i \rightarrow \infty} \mu^{*}\left(M_{i}\right)$

(3) The rhombic limit of $M$ is the point $\vec{\rho}(M)=\left(\vec{\mu}(M), \overrightarrow{\mu^{*}}(M)\right)$ in the rhombic picture.

Lemma 4.7. For $M$ a preinjective module, $\operatorname{fin}(M)>_{\mathcal{F}} \operatorname{per}(M)$ holds.

Proof. The statement is clear if $\operatorname{per}(M)=(h)$. If $\operatorname{per}(M)=\mathcal{L}$ (or $\mathcal{R})$, note that $M$ is the epimorphic image of an irreducible map given by deleting a cohook on the left (right) end of the string defining $M$. Hence, in the Greedy Algorithm, after the periodic part, the left (right) hook sequence is a non-empty but incomplete sequence. It is obtained from $\mathcal{L}(\mathcal{R})$ by reducing one of the parts and omitting all following parts. This partial sequence is the initial part of fin $(M)$.

It is clear that GR-limits given by a ray are approached from below, and GR-colimits given by a coray are approached from above. From the lemma we deduce:

Corollary 4.8. Preinjective families approach the GR-limit from above, and preprojective families approach the GR-colimit from below.

The following definition is found in [9]. We state it here for the convenience of the reader. 
Definition 4.9. (1) The take-off limit $\vec{\mu}_{T}$ is the supremum of the measures in the take-off part of the module category.

(2) The landing limit $\vec{\mu}_{L}$ the infimum of the measures in the landing part of the module category.

Following suit, we have the following.

Definition 4.10. By the homogeneous limit $\vec{\mu}_{H}$ we denote the limit of the measures of the modules in a homogeneous tube.

In conclusion, we determine from which direction the limit points in the rhombic picture are approached.

Proposition 4.11. For an indecomposable module $M$ with family $\left(M_{i}\right)$, the following statements are equivalent.

(1) $M$ is preprojective.

(2) $\operatorname{per}(M)>_{\mathcal{F}} \operatorname{fin}(M)$ and $\operatorname{per}^{*}(M)>_{\mathcal{F}}^{*} \operatorname{fin}^{*}(M)$.

(3) The points $\rho\left(M_{i}\right)$ approach the rhombic limit $\vec{\rho}(M)$ from the left.

Moreover, if $M$ is preprojective then the rhombic limit $\vec{\rho}(M)=\left(\vec{\mu}, \vec{\mu}^{*}\right)$ satisfies

$$
\vec{\mu}=\vec{\mu}_{T} \quad \text { and } \quad \vec{\mu}_{L}^{*} \leq_{\mathcal{F}}^{*} \vec{\mu}^{*} \leq_{\mathcal{F}}^{*} \vec{\mu}_{H}^{*}
$$

Proof. It is clear that (2) and (3) are equivalent. We show that (1) implies (2), the converse follows from the corresponding results for preinjective and for regular modules below. Suppose that $M$ is preprojective. Then $D M$ is preinjective, so $\operatorname{fin}(D M)>\operatorname{per}(D M)$ by Lemma 4.7. As the ordering for comeasures is the opposite of the ordering for measures, fin* $(M)<\operatorname{per}^{*}(M)$ follows.

Regarding the last assertion, each preprojective module is in the takeoff part [4, Theorem 3.3], so the GR-limit is the take-off limit $\vec{\mu}_{T}$, while for each preinjective module, the GR-limit is at most the landing limit $\vec{\mu}_{L}$. The last inequality follows from Lemma 3.4 as the GR-limit for a preinjective module is at least the homogeneous limit $\vec{\mu}_{H}$.

Similarly we have:

Proposition 4.12. For an indecomposable module $M$ with family $\left(M_{i}\right)$, the following statements are equivalent.

(1) $M$ is preinjective.

(2) $\operatorname{per}(M)<_{\mathcal{F}} \operatorname{fin}(M)$ and $\operatorname{per}^{*}(M)<_{\mathcal{F}} \operatorname{fin}^{*}(M)$.

(3) In the rhombic picture, the points $\rho\left(M_{i}\right)$ approach $\vec{\rho}(M)$ from the right.

Moreover, if $M$ is preinjective then the rhombic limit $\vec{\rho}(M)=\left(\vec{\mu}, \vec{\mu}^{*}\right)$ satisfies $\vec{\mu}^{*}=\vec{\mu}_{T}^{*}$ and $\vec{\mu}_{H} \leq_{\mathcal{F}} \vec{\mu} \leq_{\mathcal{F}} \vec{\mu}_{L}$. 
For regular modules we obtain:

Proposition 4.13. For an indecomposable module $M$ with family $\left(M_{i}\right)$, the following statements are equivalent.

(1) $M$ occurs in a tube.

(2) $\operatorname{per}(M)>_{\mathcal{F}} \operatorname{fin}(M)$ and $\operatorname{per}^{*}(M)<_{\mathcal{F}}$ fin $^{*}(M)$.

(3) In the rhombic picture, the points $\rho\left(M_{i}\right)$ approach $\vec{\rho}(M)$ from below.

ExAMPLE 4.14. We apply the above results to the two sink-two source quiver from Example 1.6. There are five projective modules, but six families of preprojective modules, corresponding to the string types $b b_{*}, b c_{*}, b e_{*}, e b_{*}, e c_{*}, e e_{*}$. The families approach the rhombic limit $\vec{\rho}$ from the left; the rhombic limit has the form $\vec{\rho}=\left(\vec{\mu}_{T}, \vec{\mu}^{*}\right)$ where $\vec{\mu}_{L}^{*} \leq_{\mathcal{F}}^{*} \vec{\mu}^{*} \leq_{\mathcal{F}}^{*} \vec{\mu}_{H}^{*}$.

Dually, the preinjective modules have string types $a a_{*}, a d_{*}, c d_{*}, d d_{*}$, $d a_{*}, c a_{*}$. They approach the rhombic limit from the right; the limit has the form $\left(\vec{\mu}, \vec{\mu}_{T}^{*}\right)$ where $\vec{\mu}_{H} \leq_{\mathcal{F}} \vec{\mu} \leq_{\mathcal{F}} \vec{\mu}_{L}$.

The remaining families consist of regular modules, they all approach the rhombic limit from below.

All families and their limits are shown in in Figure 1.

\section{Auslander-Reiten Sequences in the Rhombic Picture}

Here we present a result that further reveals the connection between Auslander-Reiten sequences and the rhombic picture. In this section, we put no condition on the underlying algebra. The following statement holds, as in Proposition 4.13.

REMARK 5.1. If $M$ is an indecomposable module in a stable tube, and $\left(M_{i}\right)$ the family of $M$, then the points $\rho\left(M_{i}\right)$ approach the rhombic limit $\vec{\rho}(M)$ from below.

Theorem 5.2. Let $0 \rightarrow A \rightarrow B_{1} \oplus B_{2} \rightarrow C \rightarrow 0$ be an AuslanderReiten sequence in a stable tube such that the middle term consists of two indecomposable summands. Then the rhombic limits of $A, B_{1}, B_{2}, C$ form a (possibly degenerate) parallelogram in the rhombic picture. Moreover, the nondegenerate sides of the parallelogram are parallel to the $\mu$ and $\mu^{*}$ axes.

Proof. We may assume that the map $A \rightarrow B_{1}$ is monic; otherwise, exchange $B_{1}$ and $B_{2}$. Now since the modules $A, B_{1}$ lie on the same ray in the tube, they have the same GR-limit. Hence, their rhombic limits 
FiguRE 1. The rhombic picture for the first example

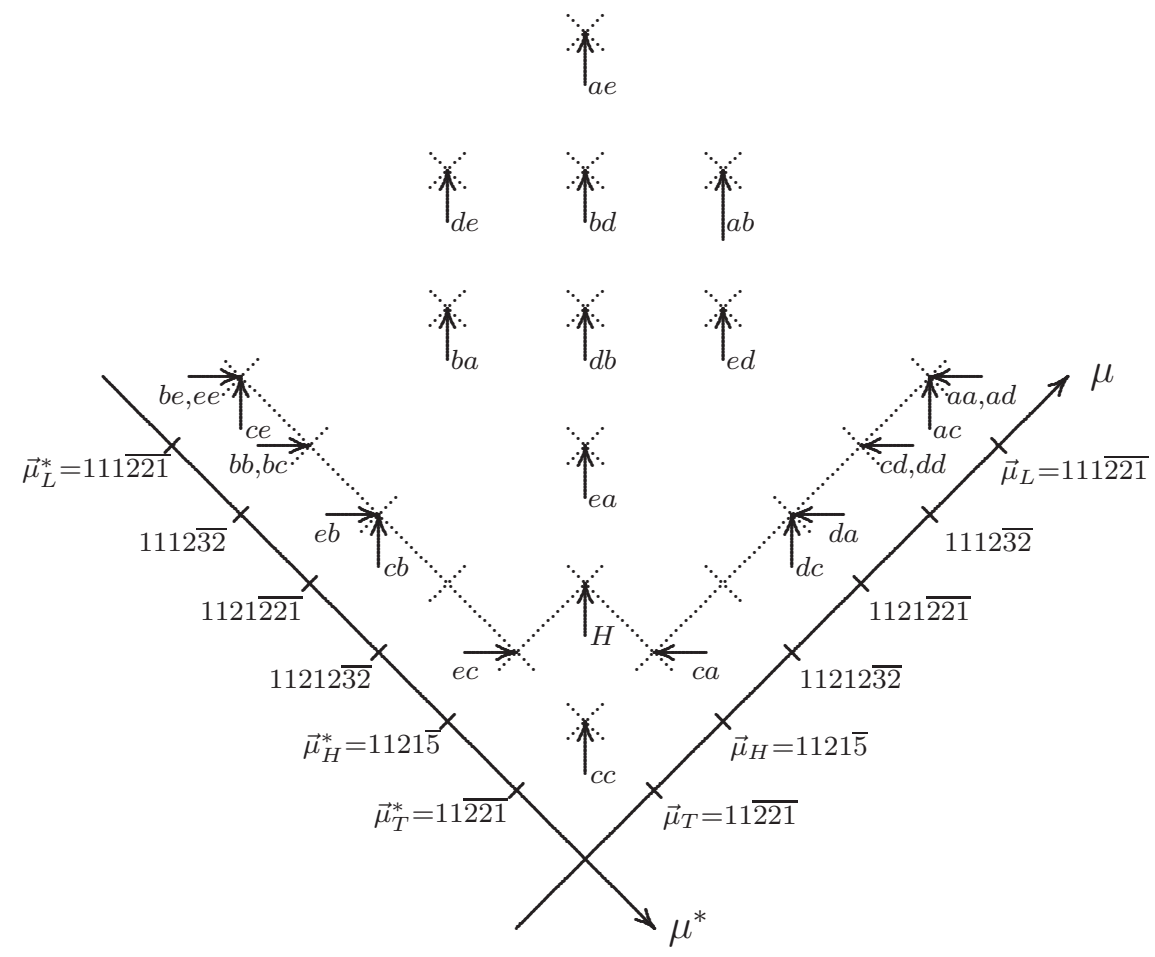

lie on a line parallel to the $\mu^{*}$ axis in the rhombic picture. The same is true for the modules $B_{2}, C$. Similarly, $A, B_{2}$ are on the same coray in the tube and, hence, they have the same GR-colimit. Thus, their rhombic limits lie on a line parallel to the $\mu$ axis in the rhombic picture. The same is true for the modules $B_{1}, C$. The result follows.

ExAMPLE 5.3. For the path algebra of the quiver studied in Example 1.6 and 4.14 there are two extended tubes. The right tube is pictured in Example 1.6. There are nine families, corresponding to the types $a b_{*}, a c_{*}, a e_{*}, c b_{*}, c c_{*}, c e_{*}, d b_{*}, d c_{*}, d e_{*}$. Note that all modules in this tube satisfy per $=221$ and per* $=221$.

The left tube pictured in Figure2 2 consists of four families corresponding to the string types $b a_{*}, b d_{*}, e a_{*}, e d_{*} ;$ all have periodic parts per $=32$ and per $^{*}=32$.

We see that each Auslander-Reiten sequence in one of the tubes gives rise to a non-degenerate parallelogram in the rhombic picture in Figure 1 . 
Figure 2. The left tube in the first example

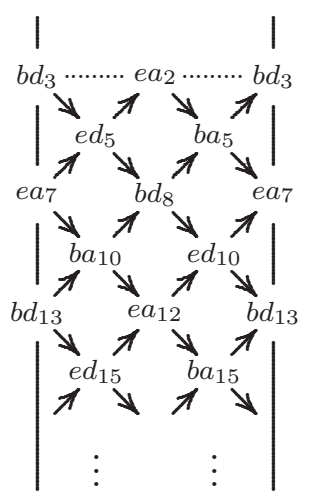

\section{The Tiling Theorem}

We return to the case where $M$ is a representation for a quiver of type $\widetilde{\mathbb{A}}_{n}$. We have seen that $M$ is regular if and only if $\operatorname{per}(M)>_{\mathcal{F}} \operatorname{fin}(M)$ and $\operatorname{per}^{*}(M)<_{\mathcal{F}}$ fin* $(M)$. We can say more.

6.1. Three kinds of tubes. As we have fixed an orientation of the quiver, we can define the left tube as the exceptional tube in which irreducible monomorphisms are given by adding a hook on the left; the right tube is similarly defined. It is well-known that all remaining tubes are homogeneous.

Proposition 6.1. An indecomposable regular module $M$ is in the left tube, in the right tube, or in a homogeneous tube if and only if the periodic part $\operatorname{per}(M)$ is $\mathcal{L}, \mathcal{R}$, or $(h)$, respectively, where $h=n+1$.

Proof. The result will follow from our investigation of the tubes in Section 6.4.

Thus, it can be read off from the pair $\left(\mu(M), \mu^{*}(M)\right)$ whether $M$ is a regular module, and if so in which tube $M$ occurs.

If the tube is exceptional, then the pair even determines the ray and the coray of $M$. To visualize this, we introduce two partial orderings.

6.2. The staircase ordering. We consider families of points in the rhombic picture such that the limits of their measures agree and are approached from below. We have seen in Chapter 4 that the families given by a ray in an exceptional tube have this property. 
Definition 6.2. For $\mu \in \mathcal{F}$, let $S(\vec{\mu})$ be the set of equivalence classes of sequences $\left(\mu_{i}, \mu_{i}^{*}\right)$ in $\mathcal{F} \times \mathcal{F}^{*}$ such that the $\mu_{i}$ are strictly increasing with limit $\vec{\mu}$ and the $\mu_{i}^{*}$ are strictly decreasing. Two sequences are equivalent if the difference set is finite.

For two sequences $\mathcal{M}=\left(\mu_{i}, \mu_{i}^{*}\right), \mathcal{M}^{\prime}=\left(\mu_{i}^{\prime}, \mu_{i}^{\prime *}\right)$ in $S(\vec{\mu})$ we define $\mathcal{M}^{\prime} \leq_{\text {stair }} \mathcal{M}$ if for almost all $n$ there is an $\ell$ such that

$$
\mu_{\ell}^{\prime} \leq_{\mathcal{F}} \mu_{n}<_{\mathcal{F}} \mu_{\ell+1}^{\prime} \quad \text { and } \mu_{\ell}^{*} \leq_{\mathcal{F}}^{*} \mu_{n}^{*} .
$$

Lemma 6.3. For each $\mu \in \mathcal{F}$, the relation $\leq_{\text {stair }}$ is a partial ordering on $S(\mu)$.

Proof. We only show anti-symmetry: Suppose $M=\left(\mu_{i}, \mu_{i}^{*}\right), M^{\prime}=$ $\left(\mu_{i}^{\prime}, \mu_{i}^{\prime *}\right)$ represent equivalence classes of sequences in $S(\mu)$ and satisfy $M^{\prime} \leq_{\text {stair }} M$ and $M \leq_{\text {stair }} M^{\prime}$. We verify that $M$ and $M^{\prime}$ are equivalent. There exist $L, N \in \mathbb{N}$ such that for each $n \geq N$ there is $\ell \geq L$ with

$$
\mu_{\ell}^{\prime} \leq_{\mathcal{F}} \mu_{n}<_{\mathcal{F}} \mu_{\ell+1}^{\prime} \quad \text { and } \quad \mu_{\ell}^{\prime *} \leq_{\mathcal{F}}^{*} \mu_{n}^{*}
$$

and such that for each $\ell \geq L$ there is $m$ with

$$
\mu_{m} \leq_{\mathcal{F}} \mu_{\ell}^{\prime}<_{\mathcal{F}} \mu_{m+1} \quad \text { and } \quad \mu_{m}^{*} \leq_{\mathcal{F}}^{*} \mu_{\ell}^{\prime *} .
$$

Note that given $n$, both $\ell$ and $m$ are uniquely determined since the sequences $\mu_{i}, \mu_{i}^{\prime}$ are strictly increasing. Since $\mu_{m} \leq_{\mathcal{F}} \mu_{n}$, we obtain $m \leq n$ and hence $\mu_{n}^{*} \leq_{\mathcal{F}}^{*} \mu_{m}^{*}$ since the sequence $\mu_{i}^{*}$ is strictly decreasing. Together with the above $\mu_{m}^{*} \leq_{\mathcal{F}}^{*} \mu_{\ell}^{*} \leq_{\mathcal{F}}^{*} \mu_{n}^{*}$ we obtain $\mu_{m}^{*}=\mu_{n}^{*}$, hence $m=n$. It follows $\mu_{n}=\mu_{\ell}^{\prime}$ and $\mu_{n}^{*}=\mu_{\ell}^{\prime *}$. We have shown that $M$ and $M^{\prime}$ differ at most by an initial segment.

EXAMPLE 6.4. As second example we consider the path algebra $k Q$ for the quiver

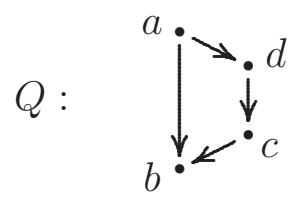

The module $c c_{1}$ is simple regular; the modules on its ray,

$$
c c_{1}, c d_{2}, c b_{4}, c c_{5}, c d_{6}, c b_{8}, c c_{9}, \ldots
$$

have the following positions in the rhombic picture. They occur in three families, which are indicated by solid lines in Figure 3. To emphasize the shape of the staircase, we rotate the picture by $-45^{\circ}$.

The families of regular modules satisfy $c d_{*} \leq_{\text {stair }} c c_{*} \leq_{\text {stair }} c b_{*}$. Note $c c_{*}$ and $c d_{*}$ approach the same limit in the rhombic picture.

By comparison, families in the preprojective component with the same rhombic limit may be incomparable with respect to $\leq_{\text {stair }}$. In Figure 4 , 
FiguRE 3. The staircase ordering for regular modules

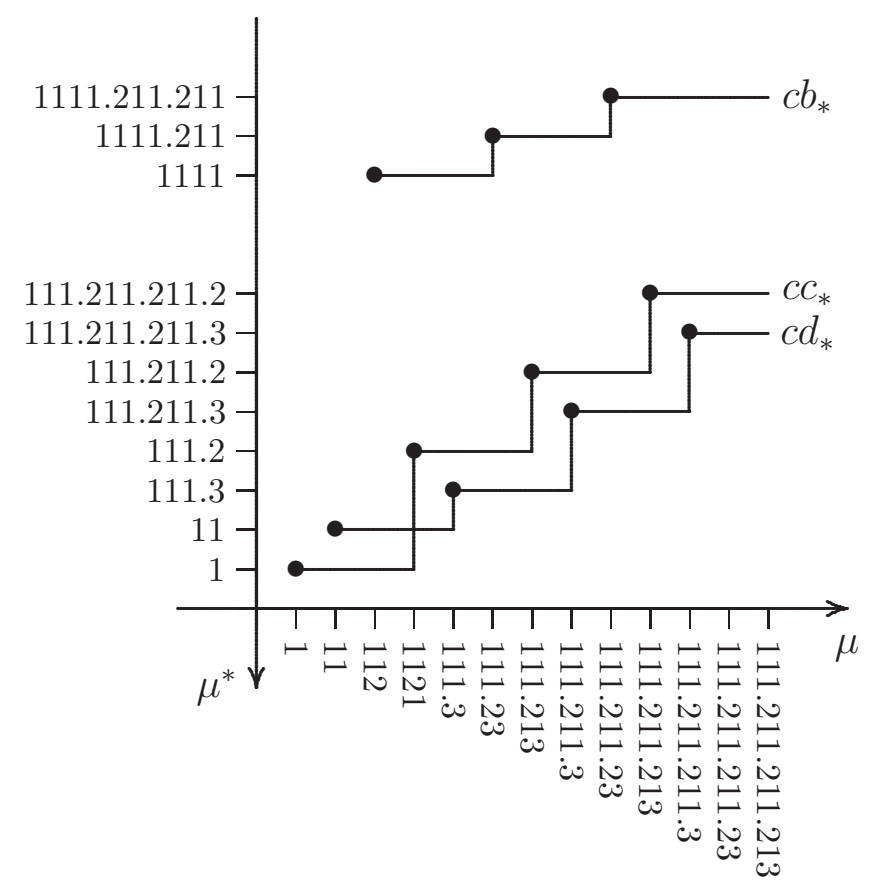

we picture one ray starting at the simple projective module $b b_{1}$. The families $b c_{*}$ and $b d_{*}$ illustrate the situation mentioned.

6.3. The ordering given by the waist-free parts. We have seen that for a regular module $M$, the periodic part $\operatorname{per}(M)$ determines the type the tube, while the multiplicity $\operatorname{mult}(M)$ specifies the position within its family. The remaining data form the waist-free part of the measure; similarly, one can define the waist-free part of the comeasure.

$$
\mathrm{wf}(M)=\operatorname{init}(M) \cdot \operatorname{fin}(M) ; \quad \mathrm{wf}^{*}(M)=\operatorname{init}^{*}(M) \cdot \mathrm{fin}^{*}(M)
$$

Note that wf $(M)$ and $\mathrm{wf}^{*}(M)$ do not depend on the representative $M$ of a family; thus they allow us to define a partial ordering for families: For two families $\left(M_{i}\right),\left(M_{j}^{\prime}\right)$ we define $\left(M_{i}\right) \leq_{\mathrm{wf}}^{*}\left(M_{j}^{\prime}\right)$ if $\mathrm{wf}^{*}\left(M_{i}\right) \leq_{\mathcal{F}}^{*}$ wf $^{*}\left(M_{j}^{\prime}\right)$ holds for modules $M_{i}$ and $M_{j}^{\prime}$.

Proposition 6.5. Consider the set $\mathcal{S}$ of families given by a ray in one of the tubes.

(1) The set $\mathcal{S}$ is totally ordered with respect to $\leq_{\text {stair }}$.

(2) The set $\mathcal{S}$ is totally ordered with respect to $\leq_{\mathrm{wf}}^{*}$.

(3) The two orderings $\leq_{\text {stair }}$ and $\leq_{\mathrm{wf}}^{*}$ are equivalent. 
FigurE 4. The staircase ordering for preprojective modules

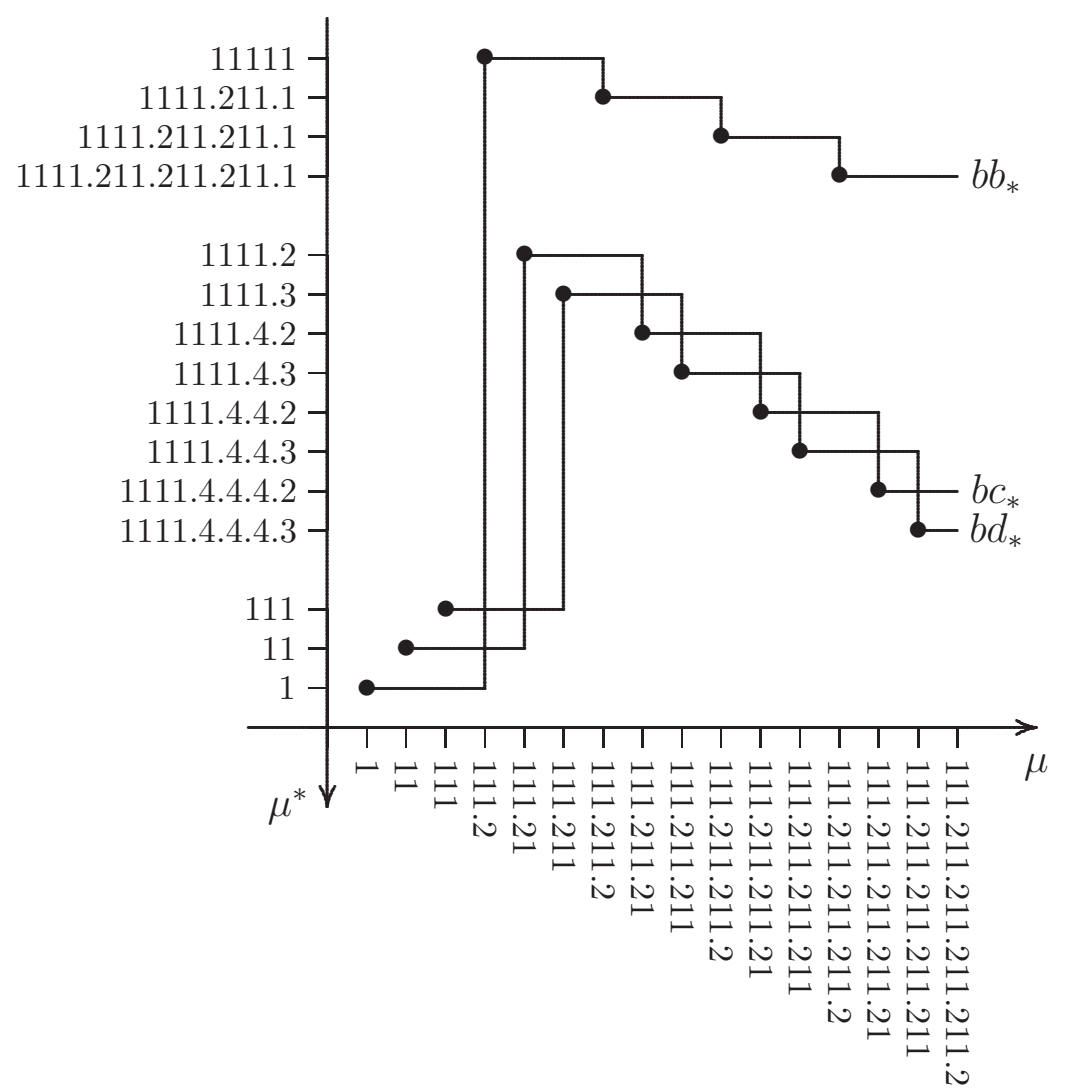

Proof. The result will follow from our discussion of the tubes in Section 6.4 .

Using the waist-free ordering we can strengthen Theorem 5.2 in the sense that the parallelograms given by Auslander-Reiten sequences are always non-degenerate.

Corollary 6.6. Let $0 \rightarrow A \rightarrow B_{1} \oplus B_{2} \rightarrow C \rightarrow 0$ be an AuslanderReiten sequence in a nonhomogeneous tube Then the rectangle with vertices

$\left(\mathrm{wf}(A), \mathrm{wf}^{*}(A)\right),\left(\mathrm{wf}\left(B_{1}\right), \mathrm{wf}^{*}\left(B_{1}\right)\right),\left(\mathrm{wf}\left(B_{2}\right), \mathrm{wf}^{*}\left(B_{2}\right)\right),\left(\mathrm{wf}(C), \mathrm{wf}^{*}(C)\right)$ in the rhombic picture is non-degenerate.

6.4. A discussion of the tubes. For each tube we now investigate how the GR-measures change along a coray. We then give the proofs for Proposition 6.1 and Proposition 6.5.

We assume first that the take-off direction is to the right, i.e. $\mathcal{L}<_{\mathcal{F}} \mathcal{R}$ holds, and then consider the case of a symmetric quiver. 
First we deal with the left tube, where irreducible monomorphisms are given by adding a hook to the left end of the string, and irreducible epimorphisms are given by deleting a cohook on the right end. Note that whenever a cohook is deleted on the right end of a string, then the sequence $\left(\rho_{i}\right)$ of right hooks used in the Greedy Algorithm ends with a part that is smaller than the corresponding part in $\mathcal{R}$. As a consequence, the starting point for the Greedy Algorithm is near the right end of the string.

For modules $M, M^{\prime}$ in two different families on the same coray in the left tube we have:

(L1) The periodic part is $\operatorname{per}(M)=\mathcal{L}$.

(L2) The initial part $\operatorname{init}(M)$ is strictly above the take-off limit $\vec{\mu}_{T}$.

(L3) Hence the GR-limit satisfies $\vec{\mu}(M)>_{\mathcal{F}} \vec{\mu}_{T}$.

(L4) The initial parts $\operatorname{init}(M), \operatorname{init}\left(M^{\prime}\right)$ have different lengths, the final parts are equal: $\operatorname{fin}(M)=\operatorname{fin}\left(M^{\prime}\right)$.

(L5) The inequalities are equivalent:

$$
\operatorname{init}(M)<_{\mathcal{F}} \operatorname{init}\left(M^{\prime}\right) \Longleftrightarrow \operatorname{wf}(M)<_{\mathcal{F}} \operatorname{wf}\left(M^{\prime}\right) \Longleftrightarrow \vec{\mu}(M)<_{\mathcal{F}} \vec{\mu}\left(M^{\prime}\right)
$$

Next we deal with the right tube, so along a ray, hooks are added on the right end of the string, and along a coray, cohooks are deleted from the left end of the string. From the discussion of the Greedy Algorithm it follows that the starting point is near the left end of the string.

For a module $M$ in the right tube we have:

(R1) The periodic part is $\operatorname{per}(M)=\mathcal{R}$.

(R2) The initial part $\operatorname{init}(M)$ is not necessarily above the take-off limit $\vec{\mu}_{T}$.

We consider the two cases given by (R2) separately:

Suppose $M, M^{\prime}$ occur in different families on the same coray, and have both initial parts above the take-off limit $\vec{\mu}_{T}$. Then the final parts fin $(M)$, fin $\left(M^{\prime}\right)$ are equal and consist only of parts of the right hook sequence $\mathcal{R}$.

(R3') The GR-limit is above the take-off limit, $\vec{\mu}_{T}<_{\mathcal{F}} \vec{\mu}(M)$.

$\left(\mathrm{R} 4^{\prime}\right)$ The initial parts $\operatorname{init}(M)$, init $\left(M^{\prime}\right)$ have different lengths, and the final parts are equal, $\operatorname{fin}(M)=\mathrm{fin}\left(M^{\prime}\right)$.

$\left(\mathrm{R} 5^{\prime}\right)$ The inequalities are equivalent:

$$
\operatorname{init}(M)<_{\mathcal{F}} \operatorname{init}\left(M^{\prime}\right) \Longleftrightarrow \operatorname{wf}(M)<_{\mathcal{F}} \mathrm{wf}\left(M^{\prime}\right) \Longleftrightarrow \vec{\mu}(M)<_{\mathcal{F}} \vec{\mu}\left(M^{\prime}\right)
$$

Now assume that $M, M^{\prime}$ occur in different families on the same coray in the right tube such that both have initial parts below the take-off limit $\vec{\mu}_{T}$. Then $\operatorname{fin}(M)$ and $\operatorname{fin}\left(M^{\prime}\right)$ are obtained by the Greedy Algorithm 
from the same set of parts from $\mathcal{R}$, possibly from some parts from $\mathcal{L}$, and from one short left hook.

$\left(\mathrm{R} 3^{\prime \prime}\right)$ The GR-limit is the take-off limit, $\vec{\mu}_{T}=\vec{\mu}(M)$.

$\left(\mathrm{R} 4^{\prime \prime}\right)$ The initial parts are equal, $\operatorname{init}(M)=\operatorname{init}\left(M^{\prime}\right)$; the final parts fin $(M)$, fin $\left(M^{\prime}\right)$ have different lengths; their length difference is less than $h$.

$\left(\mathrm{R} 5^{\prime \prime}\right)$ The inequalities are equivalent:

$$
\ell \operatorname{fin}(M)>\ell \operatorname{fin}\left(M^{\prime}\right) \Longleftrightarrow \operatorname{fin}(M)<_{\mathcal{F}} \operatorname{fin}\left(M^{\prime}\right) \Longleftrightarrow \operatorname{wf}(M)<_{\mathcal{F}} \operatorname{wf}\left(M^{\prime}\right)
$$

Let $M$ be a quasi-simple homogeneous module (see [8]), and let $M^{\prime}$ be another module in the family of $M$, which is just the tube of $M$.

(H1) The periodic part is $\operatorname{per}\left(M^{\prime}\right)=(h)$.

(H2) The initial part init $\left(M^{\prime}\right)$ equals $\mu(M)$.

(H3) The GR-limit satisfies $\vec{\mu}\left(M^{\prime}\right)>_{\mathcal{F}} \vec{\mu}_{T}$.

It remains to deal with the case where $Q$ is a symmetric quiver, so there is a non-trivial symmetry operation on $Q$, which gives rise to a self-equivalence of $\bmod K Q$ that permutes the two tubes. We obtain:

(S0) The set of points in the rhombic picture corresponding to the modules in the left tube is identical with the set of points for the right tube.

(S1-5) For both tubes, the statements (L1)-(L5) hold.

We can now complete the remaining proofs.

Proof of Proposition 6.1. The periodic parts of the left tube, the right tube, and a homogeneous tube are $\mathcal{L}, \mathcal{R}$, and $(h)$, according to (L1), (R1), and (H1). As indicated before (S1), in the symmetric case the GR-data cannot distinguish the two exceptional tubes; for each the periodic part is $\mathcal{L}=\mathcal{R}$.

Proof of Proposition 6.5. Fix a ray in one of the exceptional tubes, and let $\left(M_{i}\right)$ and $\left(M_{i}^{\prime}\right)$ be two families on the ray. Put $\mu_{i}=\mu\left(M_{i}\right)$, $\mu_{i}^{\prime}=\mu\left(M_{i}^{\prime}\right), \mu_{i}^{*}=\mu^{*}\left(M_{i}\right), \mu_{i}^{\prime *}=\mu^{*}\left(M_{i}^{\prime}\right)$. As the measures along the ray increase monotonically, say towards $\vec{\mu}$, the families $\left(M_{i}\right),\left(M_{i}^{\prime}\right)$ have the same GR-limit $\vec{\mu}=\lim _{i} \mu_{i}=\lim _{i} \mu_{i}^{\prime}$. Since the modules are regular, the periodic part is less than the final part, so within each family the GR-comeasures are strictly decreasing. Hence the families given by the ray can be compared in $\mathcal{S}(\vec{\mu})$ with respect to the orderings $\leq_{\text {stair }}$ and $\leq_{\mathrm{wf}}^{*}$.

(1) For the first part of the Proposition, we have to show that for the sequences $\mathcal{M}=\left(\mu_{i}, \mu_{i}^{*}\right), \mathcal{M}^{\prime}=\left(\mu_{i}^{\prime}, \mu_{i}^{\prime *}\right)$ either $\mathcal{M} \leq_{\text {stair }} \mathcal{M}^{\prime}$ or $\mathcal{M}^{\prime} \leq_{\text {stair }}$ 
$\mathcal{M}$ holds. Note that $\mu_{\ell}^{\prime} \leq \mu_{n}<\mu_{\ell+1}^{\prime}$ if and only if $\operatorname{dim} M_{\ell}^{\prime} \leq \operatorname{dim} M_{n}<$ $\operatorname{dim} M_{\ell}^{\prime}+h$ if and only if $\operatorname{dim} D M_{\ell}^{\prime} \leq \operatorname{dim} D M_{n}<\operatorname{dim} D M_{\ell}^{\prime}+h$. The statement is clear if $\lim _{n} \mu_{n}^{*} \neq \lim _{\ell} \mu_{\ell}^{\prime *}$. Suppose $\ell, n$ satisfy the condition $\mu_{\ell}^{\prime} \leq \mu_{n}<\mu_{\ell+1}^{\prime}$. Since the modules $M=D M_{n}, M^{\prime}=$ $D M_{\ell}^{\prime}$ lie on the same coray, we may use the above results. In the situations (L4) and (R4'), the GR-comeasures $\mu_{n}^{*}$ and $\mu_{\ell}^{\prime *}$ differ in their initial parts, and hence the limits are different. The only possibility for above limits to be equal is the situation in $\left(\mathrm{R} 4^{\prime \prime}\right)$ where the initial and periodic parts are equal, and the final parts differ in their lengths. We distinguish two cases:

First assume $\operatorname{dim} M^{\prime} \leq \operatorname{dim} M<\operatorname{dim} M^{\prime}+h$ and $\ell \operatorname{fin}(M)>\ell \operatorname{fin}\left(M^{\prime}\right)$. Then by $\left(\mathrm{R} 5^{\prime \prime}\right)$, $\operatorname{fin}(M)<_{\mathcal{F}} \operatorname{fin}\left(M^{\prime}\right)$, so $\mu_{n}^{*}>_{\mathcal{F}}^{*} \mu_{\ell}^{\prime *}$. Thus, by definition, $\mathcal{M}^{\prime} \leq_{\text {stair }} \mathcal{M}$.

Now assume $\operatorname{dim} M^{\prime} \leq \operatorname{dim} M<\operatorname{dim} M^{\prime}+h$ and $\ell \operatorname{fin}\left(M^{\prime}\right)>\ell \operatorname{fin}(M)$. Let $M^{\prime+}=D M_{\ell+1}^{\prime}$ be the next module in the family, so $\operatorname{dim} M \leq$ $\operatorname{dim} M^{\prime+}<\operatorname{dim} M+h$ holds. By $\left(\mathrm{R} 4^{\prime \prime}\right)$, the length of the final parts of $M$ and $M^{\prime+}$ differs by less than $h$. It follows that there is an $m \in \mathbb{N}$ such that

$$
\begin{aligned}
\mu(M) & =\operatorname{init}(M) \cdot \operatorname{per}(M)^{m+1} \cdot \operatorname{fin}(M) \\
\mu\left(M^{\prime}\right) & =\operatorname{init}(M) \cdot \operatorname{per}(M)^{m} \cdot \operatorname{fin}\left(M^{\prime}\right) \\
\mu\left(M^{\prime+}\right) & =\operatorname{init}(M) \cdot \operatorname{per}(M)^{m+1} \cdot \operatorname{fin}\left(M^{\prime}\right)
\end{aligned}
$$

hold. Again by $\left(\mathrm{R} 5{ }^{\prime \prime}\right), \operatorname{fin}\left(M^{\prime}\right)<_{\mathcal{F}} \operatorname{fin}(M)$, so $\mu_{\ell+1}^{\prime *}>_{\mathcal{F}}^{*} \mu_{n}^{*}$. Thus, by definition, $\mathcal{M}^{\prime} \geq_{\text {stair }} \mathcal{M}$.

(2) Clearly, different families on a coray have different waist-free parts. As a consequence, $\leq_{\mathrm{wf}}^{*}$ is a total ordering for the families on a ray in an exceptional tube.

(3) The staircase ordering is as follows: $\mathcal{M}^{\prime} \leq_{\text {stair }} \mathcal{M}$ if $\lim _{i} \mu_{i}^{\prime *} \leq_{\mathcal{F}}^{*}$ $\lim _{i} \mu_{i}^{*}$, and in case the limits are equal, if $\ell \operatorname{fin}\left(D M_{j}^{\prime}\right) \leq \ell$ fin $\left(D M_{i}\right)$ holds for $i, j$ suitably large. The condition on the limits is equivalent to $\operatorname{init}\left(D M_{j}^{\prime}\right) \geq_{\mathcal{F}} \operatorname{init}\left(D M_{i}\right)$, the condition on the lengths is equivalent to fin $\left(D M_{j}^{\prime}\right) \geq_{\mathcal{F}}$ fin $\left(D M_{i}\right)$ by $\left(\mathrm{R} 5^{\prime \prime}\right)$. In conclusion, $\mathcal{M}^{\prime} \leq_{\text {stair }} \mathcal{M}$ holds if and only if $\operatorname{wf}\left(D M_{j}^{\prime}\right) \geq_{\mathcal{F}} \operatorname{wf}\left(D M_{i}\right)$, which is equivalent to $\mathcal{M}^{\prime} \leq_{\text {wf }}^{*} \mathcal{M}$.

\subsection{The Tiling Theorem.}

Definition 6.7. We say that a quiver $Q$ of type $\widetilde{\mathbb{A}}_{n}$ has a unique widest valley if in each exceptional tube, the same sink, up to the periodic shift, may be chosen as Greedy Algorithm starting point for all modules $M$ of sufficiently large dimension. 
Dually, $Q$ has a unique widest hill if in each exceptional tube the same sink in $Q^{\mathrm{op}}$, up the shift, may be chosen as Greedy Algorithm starting point for each module $D M$ where $M$ is a sufficiently large module in the tube.

REMARK 6.8. The conditions in the definition are satisfied if $Q$ has a valley and a hill that are at least 2 units wider than any other valley and hill, respectively. If $Q$ has several valleys of maximal size, the starting point will jump among their sinks. If the difference in size is just 1 unit, a jump among different sinks may occur. For the convenience of the reader, we repeat the two-sink two-source example given earlier.

EXAMPLE 6.9. We see that the two-sink, two-source $\widetilde{\mathbb{A}}_{4}$ quiver from Example 1.6 has both a unique widest valley and a unique widest hill.

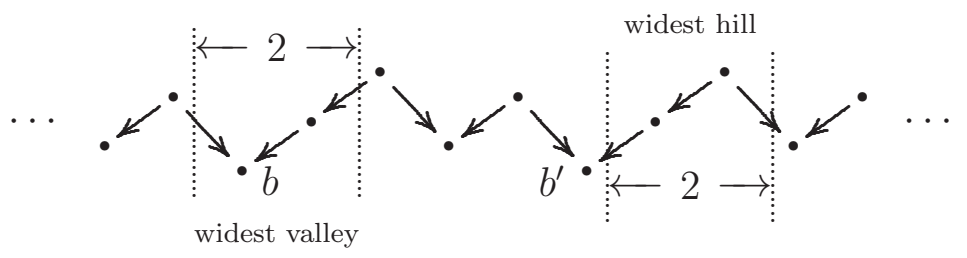

With this notion, we can add the following observation to our discussion of the tubes.

REMARK 6.10. Suppose $Q$ has a unique widest valley and assume that the modules $M, M^{\prime}$ occur on the same coray in an exceptional tube for $k Q$. Then $\operatorname{init}(M)<_{\mathcal{F}} \operatorname{init}\left(M^{\prime}\right)$ if and only if $\ell \operatorname{init}(M)>\ell \operatorname{init}\left(M^{\prime}\right)$. Here we denote by $\ell$ the sum of the parts of a finite sequence in $\mathcal{F}$.

We now restate our final result, The Tiling Theorem.

THEOREM 6.11. Suppose the quiver $Q$ of type $\widetilde{\mathbb{A}}_{n}$ has a unique widest valley and a unique widest hill. Then for each tube, the system of limits in the rhombic picture provides a tiling for the tube.

A technical lemma is needed before we can commence the proof of the theorem.

LEMMA 6.12. Suppose all sufficiently large modules on a coray in a non-homogeneous tube have the same Greedy Algorithm starting point, up to the periodic shift. Then the ordering of the waist-free parts of the families agrees with the sequence in which they occur along the coray.

Proof. Along a coray, the lengths of the waist-free parts follow a sawtooth pattern as they decrease until the starting point "jumps". We 
have seen in the discussion of the tubes that as the lengths of the waistfree parts decrease, their measures increase in $\mathcal{F}$. Thus, by choosing a suitable first family on the coray, the ordering of the families agrees with the ordering of their the waist-free parts.

Proof of the Tiling Theorem 6.11. We have already seen that the ordering of families by their waist-free parts agrees with the staircase ordering which we picture as a left-right ordering in the rhombic picture. Using the lemma and its dual version we obtain the result.

\subsection{Examples.}

ExAmple 6.13. The quiver $Q$ considered in Example 1.6 is symmetric with respect to rotation by $\pi$, so the take-off direction of $Q$, and the take-off direction of $Q^{\text {op }}$ are both to the right.

The left tube pictured in Figure 2 consists of the regular modules with with periodic parts per $=32$ and per* $=32$. The GR-limits in the rhombic picture in Figure 1 are above the take-off limit and their GRcolimits are below the take-off colimit, hence the limit points are determined uniquely by the inital parts (see (L2), (L4) above).

The right tube is given by per $=221$, per* $=221$. Here the take-off limit and the take-off colimits are attained, but only by one family on each coray and each ray, respectively. So the limit points are still determined uniquely by the inital parts (see (R2), (R4'), (R3"), (R4') above). The picture in the introduction shows how the system of limit points in the rhombic picture corresponds to a tiling of the tube.

EXAMPLE 6.14. We now discuss briefly the rhombic picture in Figure 5 for the one sink-one source quiver in Example 6.4. Again, the take-off direction and the take-off codirection are both to the right. Here, the left tube consists only of one family, $b a_{*}$.

The right tube, which consists of the nine families $a b_{*}, a c_{*}, a d_{*}, c b_{*}, c c_{*}$, $c d_{*}, d b_{*}, d c_{*}, d d_{*}$, has the property that on each ray, the comeasures for two of three families approach the take-off colimit, and on each coray, the measures for two families approach the take-off limit. Thus in the rhombic picture, there are only four limit points for nine families.

Along the ray $c c_{1} \rightarrow c d_{2} \rightarrow c b_{4} \rightarrow c c_{5} \rightarrow \cdots$, the families $c c_{*}, c d_{*}$ have the same colimits, but we can use the staircase ordering to refine the colimit by taking also the direction from where the colimit is approached into account, as we have seen in Example 6.4. Using this refinement, the system of limits in the rhombic picture produces a tiling of the exceptional tube pictured in Figure 6 . 
Figure 5. The rhombic picture for the second example

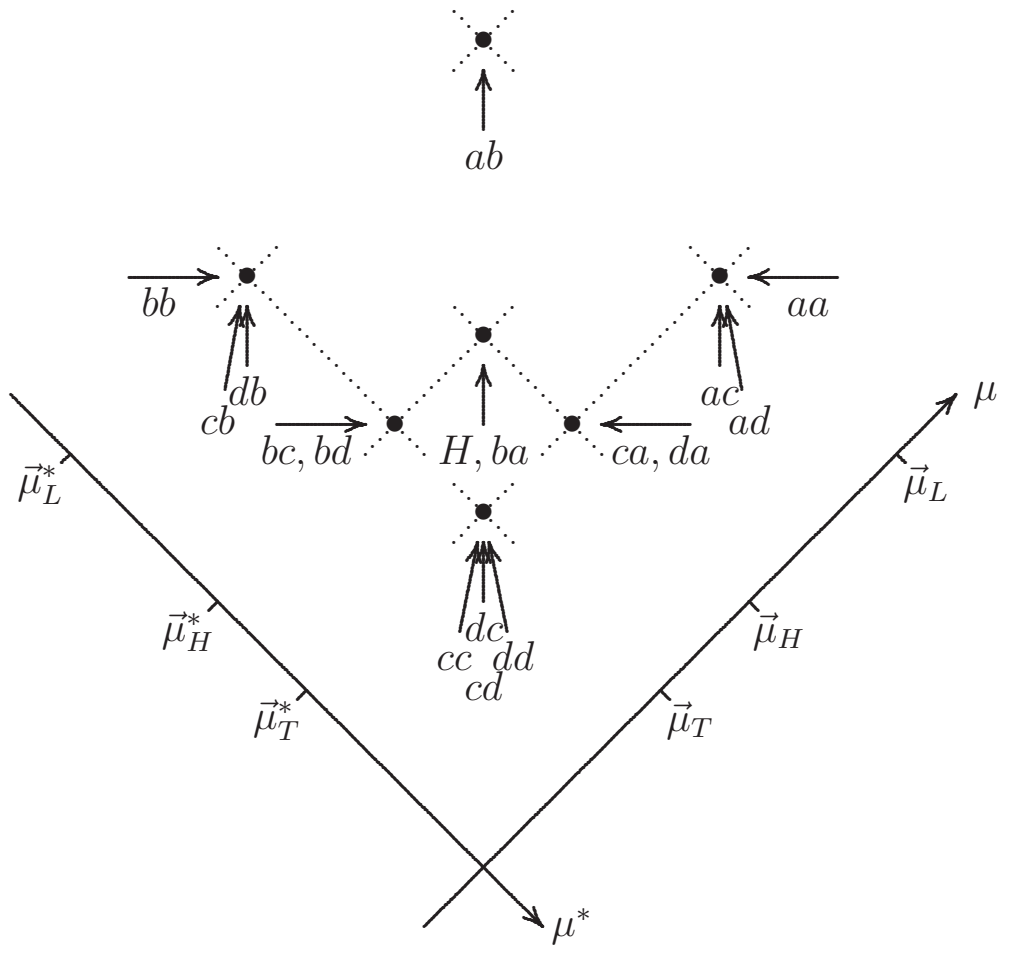

Figure 6. The right tube in the second example

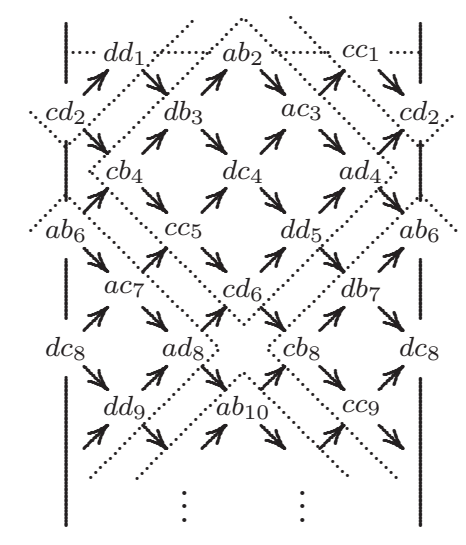

\section{ACKnowledgements}

The first named author wishes to thank Manhattan College for the invitation to two monthlong visits in the summers of 2010 and 2011 . The 
project was initiated when the second named author visited Florida Atlantic University during her sabbatical leave from Manhattan College in the spring of 2010. She is grateful to both institutions for their generosity and hospitality. Both authors appreciate the helpful suggestions offered by the referee.

\section{REFERENCES}

[1] I. Assem, D. Simson, A. Skowroński, Elements of the Representation Theory of Associative Algebras 1: Techniques of Representation Theory, Cambridge Univ. Press, Cambridge, 2006.

[2] M. Auslander, I. Reiten, S. Smalø, Representation theory of Artin algebras, Cambridge Univ. Press, Cambridge, 1995.

[3] M. C. R. Butler and C. M. Ringel, Auslander-Reiten sequences with few middle terms and applications to string algebras, Comm. Algebra 15 (1987), 145-179.

[4] B. Chen, The Gabriel-Roiter Measure for $\widetilde{\mathbb{A}}_{n}$, J. Algebra 320 (2008), 28912906.

[5] B. Chen, Comparison of AuslanderReiten theory and Gabriel-Roiter measure approach to the module categories of tame hereditary algebras, Comm. Algebra 36 (2008), 4186-4200.

[6] N. V. Dung, D. Simson, The Gabriel-Roiter measure for right pure semisimple rings, Algebr. Represent. Theory 11 (2008), 407-424.

[7] H. Krause, Maps between tree and band modules, J. Algebra 137 (1991), 186194.

[8] C. M. Ringel, Tame Algebras and Integral Quadratic Forms, Lecture Notes in Math. 1099, Springer-Verlag, 1984.

[9] C. M. Ringel, The Gabriel-Roiter measure, Bull. Sci. Math. 129 (2005), 726748.

[10] C. M. Ringel, Gabriel-Roiter inclusions and Auslander-Reiten theory, J. Algebra 324 (2010), 3579-3590. 